\title{
Photoresponse enhancement in the near infrared wavelength range of ultrathin amorphous silicon photosensitive devices by integration of silver nanoparticles
}

Etienne Moulin, Peiqing Luo, Bart Pieters, Joachim Sukmanowski, Joachim Kirchhoff, Wilfried Reetz, Thomas Müller, Reinhard Carius, Francois-Xavier Royer, and Helmut Stiebig

Citation: Appl. Phys. Lett. 95, 033505 (2009);

View online: https://doi.org/10.1063/1.3157264

View Table of Contents: http://aip.scitation.org/toc/apl/95/3

Published by the American Institute of Physics

\section{Articles you may be interested in}

Enhanced semiconductor optical absorption via surface plasmon excitation in metal nanoparticles Applied Physics Letters 86, 063106 (2005); 10.1063/1.1855423

Long-range absorption enhancement in organic tandem thin-film solar cells containing silver nanoclusters Journal of Applied Physics 96, 7519 (2004); 10.1063/1.1812589

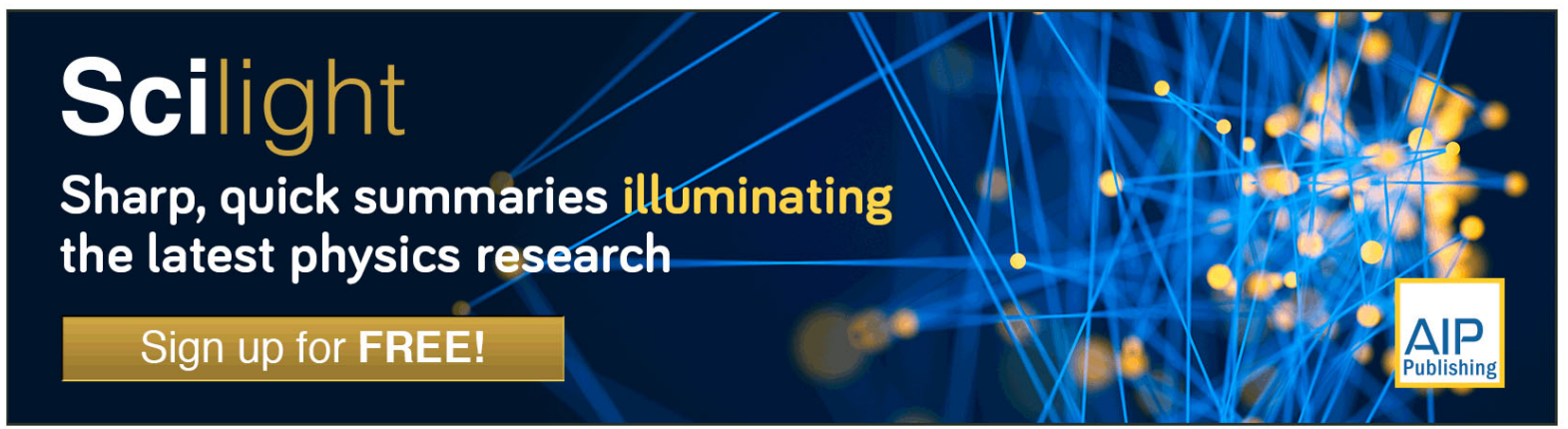




\title{
Photoresponse enhancement in the near infrared wavelength range of ultrathin amorphous silicon photosensitive devices by integration of silver nanoparticles
}

\author{
Etienne Moulin, ${ }^{1, a)}$ Peiqing Luo, ${ }^{1}$ Bart Pieters, ${ }^{1}$ Joachim Sukmanowski, ${ }^{2}$ \\ Joachim Kirchhoff, ${ }^{1}$ Wilfried Reetz, ${ }^{1}$ Thomas Müller, ${ }^{1}$ Reinhard Carius, ${ }^{1}$ \\ Francois-Xavier Royer, ${ }^{3}$ and Helmut Stiebig ${ }^{4}$ \\ ${ }^{1}$ IEF5 Photovoltaik, Forschungszentrum Jülich, 52425 Jülich, Germany \\ ${ }^{2}$ Institute of Experimental Physics, Saarland University, Postfach 151150, D-66041 Saarbruecken, Germany \\ ${ }^{3}$ Laboratoire de Physique des Milieux Denses (LPMD), Université de Metz, 1 Bd Dominique François \\ ARAGO, F-57078 Metz, France \\ ${ }^{4}$ Malibu GmbH \& Co. KG, Boettcherstrasse 7, D-33609 Bielefeld, Germany
}

(Received 19 March 2009; accepted 2 June 2009; published online 21 July 2009)

\begin{abstract}
We have investigated the contribution of localized surface plasmon polaritons (LSPPs) in silver nanoparticles with radii smaller than $20 \mathrm{~nm}$ to the photocurrent of ultrathin photosensitive devices based on amorphous silicon. An increased light absorption and an enhanced photocurrent are found for wavelengths between $600 \mathrm{~nm}$ and $1150 \mathrm{~nm}$ in presence of nanoparticles. As amorphous silicon absorbs light efficiently only at wavelengths up to $750 \mathrm{~nm}$, the increased photocurrent in the near infrared range is explained in terms of LSPP-induced photoemission of electrons within and in close vicinity of the nanoparticles. (C) 2009 American Institute of Physics. [DOI: 10.1063/1.3157264]
\end{abstract}

Metal nanoparticles (NPs) show resonances in their absorption and scattering spectra, denoted as localized surface plasmon polariton (LSPP) resonances, which are a consequence of the collective oscillation of the free electrons of the metal NP. ${ }^{1,2}$ Several authors have made use of the light scattering (amplified at the LSPP resonances) of large metal NPs (with radii $R$ between 25 and $150 \mathrm{~nm}$ ) to improve the light absorption of crystalline and amorphous silicon $(a-\mathrm{Si}: \mathrm{H})$ solar cells. ${ }^{3-6}$ The optical response of small metal NPs $(R<20 \mathrm{~nm})$ is characterized by a single LSPP resonance [dipolar surface plasmon polariton resonance]. Under these conditions the LSPP resonance is accompanied by a strong enhancement of the electromagnetic field inside and in close vicinity of the NPs. ${ }^{1,2}$ Enhanced photovoltaic conversion efficiencies of organic and dye-sensitized thin-film solar cells containing small metal NPs have already been demonstrated. $^{7-9}$ The observed increase in photocurrent is explained in terms of (i) improved light absorption in the active layer of the cell, due to the enhanced electromagnetic field in the vicinity of the NPs at the LSPP resonance or (ii) photoexcitation of electrons from the NPs to their surrounding. The positive contribution of surface plasmon polaritons (SPPs) on the photocurrent of inorganic semiconductor/metal Schottky junctions has also been reported by using the method of attenuated total reflection. ${ }^{10}$ Under these conditions, the enhanced photocurrent is explained in terms of photoexcitation of electrons from the metal surface to the semiconductor at the SPP resonance.

In order to investigate the local contribution of small metal NPs for thin-film silicon solar cell applications, $\mathrm{Ag}$ NPs of various sizes $(R<20 \mathrm{~nm})$ were deposited at the transparent conductive oxide (TCO)- $i$ interface of ultrathin $a$-Si: $\mathrm{H}$ photosensitive devices in the $\mathrm{Ag} / \mathrm{TCO} / i / p / \mathrm{TCO}$ and $\mathrm{Ag} / \mathrm{TCO} / i / n / \mathrm{TCO}$ configurations [see Fig. 1(a)]. Under these conditions, the NPs are in direct contact with the active

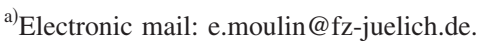

layer (the $i$-layer) of the device. The NPs were prepared by thermal evaporation of a thin Ag film at a pressure of approximately $10^{-5}$ mbar and a postannealing treatment in vacuum at a temperature of $180{ }^{\circ} \mathrm{C}$ for $90 \mathrm{~min}$. In order to obtain NPs of various sizes the thickness of the Ag film was varied between 2 and $5 \mathrm{~nm}$. The Ag bulk layer of the glass/ $\mathrm{Ag} / \mathrm{TCO}$ back reflector and the $80 \mathrm{~nm}$ thick back- and frontTCO films were deposited by sputtering at room temperature. The $20 \mathrm{~nm}$ thick $a-\mathrm{Si}: \mathrm{H} i-, n$-, and $p$-layers were deposited by plasma enhanced chemical vapor deposition.

Figure 1(b) shows a SEM (scanning electron microscope) picture of a $2 \mathrm{~nm} \mathrm{Ag}$ film on glass/Ag/TCO after annealing treatment at $180{ }^{\circ} \mathrm{C}$ for $90 \mathrm{~min}$. Well separated NPs with a broad distribution of sizes and an average radius of $10 \mathrm{~nm}$ are obtained. By increasing the thickness of the $\mathrm{Ag}$ film, the average radius of the NPs increases. For $5 \mathrm{~nm} \mathrm{Ag}$, NPs with an average radius of around $20 \mathrm{~nm}$ are formed after annealing (not shown). The influence of the NPs on the re-

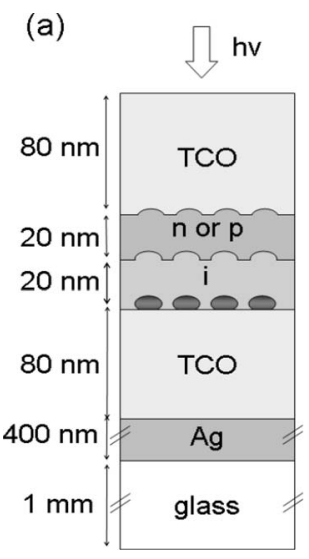

(b)

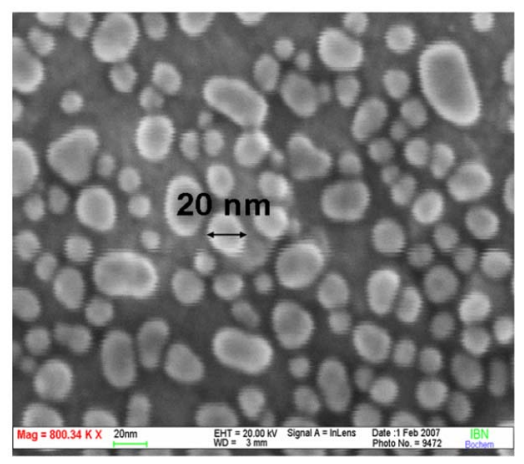

FIG. 1. (Color online) (a) Schematic drawing of the investigated $a-\mathrm{Si}: \mathrm{H}$ devices in the $\mathrm{Ag} / \mathrm{TCO} / i / n / \mathrm{TCO}$ or $\mathrm{Ag} / \mathrm{TCO} / i / p / \mathrm{TCO}$ configuration. The NPs are positioned at the $\mathrm{TCO} / i$ interface. (b) $\mathrm{SEM}$ picture of a $2 \mathrm{~nm} \mathrm{Ag}$ film on glass/Ag/TCO after annealing treatment at $180{ }^{\circ} \mathrm{C}$ for $90 \mathrm{~min}$. 

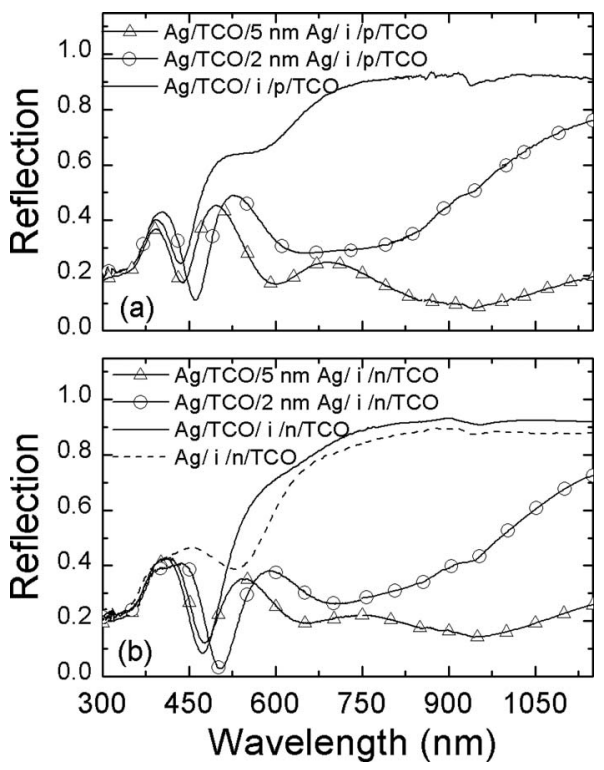

FIG. 2. Reflection measurements of (a) $\mathrm{Ag} / \mathrm{TCO} /(\mathrm{NPs}) / i / p / \mathrm{TCO}$ and (b) $\mathrm{Ag} / \mathrm{TCO} /(\mathrm{NPs}) / i / n / \mathrm{TCO}$ devices with and without nanoparticles resulting from the thermal evaporation of 2 and $5 \mathrm{~nm} \mathrm{Ag}$ films.

flectivity of the $\mathrm{Ag} / \mathrm{TCO} / i / p / \mathrm{TCO}$ and $\mathrm{Ag} / \mathrm{TCO} / i / n / \mathrm{TCO}$ devices is shown in Figs. 2(a) and 2(b), respectively. Independent of the device configuration, the layer stacks with $\mathrm{Ag}$ NPs show a strong decrease in the reflectivity for wavelengths $\lambda$ larger than $500 \mathrm{~nm}$ in comparison to the layer stacks without NPs, which is ascribed to LSPP absorption of the NPs. For thicker evaporated Ag films, the minimum in reflectivity becomes broader and is redshifted due to the larger sizes of the obtained NPs. ${ }^{11,12}$ We assume that the broad minimum in the reflectivity is due to (i) the presence of the $\mathrm{Ag}$ back reflector, which leads to interferences that partly mask the LSPP resonance and (ii) a broadening of the LSPP resonance originating from the large distribution in shapes and sizes of the NPs.

Figures 3(a) and 3(b) show the quantum efficiency (QE) at $0 \mathrm{~V}$ of devices in the $\mathrm{Ag} / \mathrm{TCO} /(\mathrm{NP}) i / p / \mathrm{TCO}$ and

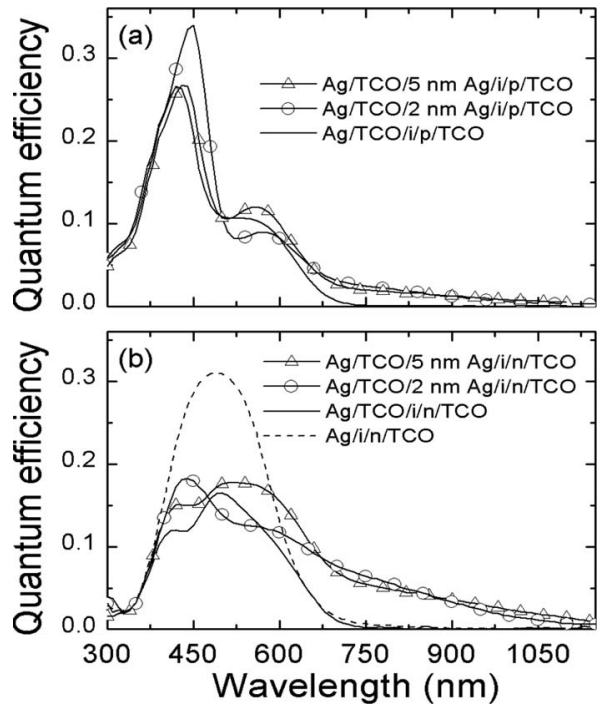

FIG. 3. QE of (a) $\mathrm{Ag} / \mathrm{TCO} /(\mathrm{NPs}) / i / p / \mathrm{TCO}$ and (b) $\mathrm{Ag} / \mathrm{TCO} /$ (NPs) $/ i / n / \mathrm{TCO}$ devices with and without NPs resulting from the thermal evaporation of 2 and $5 \mathrm{~nm} \mathrm{Ag}$ films. For comparison, the QE of the bulk- $\mathrm{Ag} / i / n / \mathrm{TCO}$ device is also shown.

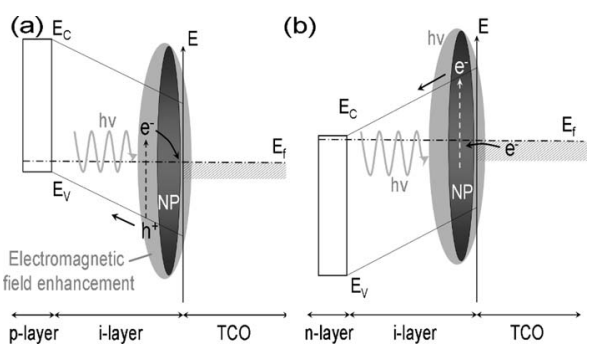

FIG. 4. Illustration of possible photoemission processes occurring in the (a) $\mathrm{Ag} / \mathrm{TCO} /(\mathrm{NPs}) / i / p / \mathrm{TCO}$ and (b) $\mathrm{Ag} / \mathrm{TCO} /(\mathrm{NPs}) / i / n / \mathrm{TCO}$ devices. Photoexcitation from states inside and in the vicinity of the NP at the LSPP resonance, charge separation, and transport processes are depicted. The symbols $e^{-}$and $h^{+}$represent electrons and holes, respectively.

$\mathrm{Ag} / \mathrm{TCO} /(\mathrm{NPs}) i / n / \mathrm{TCO}$ configuration, respectively. In the short wavelength range, the $\mathrm{QE}$ of the devices with and without NPs is nearly identical. For $\lambda>600 \mathrm{~nm}$, the photosensitive devices containing NP show an enhanced photocurrent compared to the devices without NPs. For $\lambda>750 \mathrm{~nm}$ no photocurrent is measured for the devices without NPs. In this wavelength range, $a-\mathrm{Si}: \mathrm{H}$ does not absorb light efficiently, due to its band gap of around $1.7 \mathrm{eV}$. However, a photocurrent is measured in the near infrared range (NIR) for devices with NP. The broad enhancement of the photocurrent correlates with the broad reflectivity minimum found in presence of NPs. By increasing the Ag film thickness from 2 to $5 \mathrm{~nm}$, and consequently the average size of the resulting NPs, the contribution of the photocurrent slightly shifts to longer wavelengths. Raman measurements of devices containing Ag NPs show a broadband centered at $480 \mathrm{~cm}^{-1}$, which is characteristic of $a-\mathrm{Si}: \mathrm{H}$ (not shown). However no Raman peak at $520 \mathrm{~cm}^{-1}$, characteristic of microcrystalline silicon, is found in the Raman spectrum. Therefore, we conclude that the Ag NPs does not induce the crystallization of the $a-\mathrm{Si}: \mathrm{H}$. Thus, the gain in the photocurrent observed for $\lambda>750 \mathrm{~nm}$ cannot be explained by an improved light absorption due to an alteration of the $a-\mathrm{Si}: \mathrm{H}$. We have carefully checked whether the enhanced photocurrent is due to a temperature effect resulting from enhanced light absorption in the NPs at the LSPP resonance. The photocurrent of the device without NPs increases by enhancing the device temperature. However even at $100{ }^{\circ} \mathrm{C}$ the photocurrent of the device without NPs remains much lower than the photocurrent of the device with NPs measured at room temperature. The temperature of the device is required to be far above $100{ }^{\circ} \mathrm{C}$ in order to account for the measured photocurrent. We proved that the open circuit voltage was almost identical for 532 and $897 \mathrm{~nm}$ when the same generation rate (short circuit current) was used. It is therefore concluded that the mechanism at these two wavelengths is the same and that a photovoltaic effect and no other effect such as thermopower is involved. Since $a-\mathrm{Si}: \mathrm{H}$ absorbs light efficiently only at wavelengths up to $750 \mathrm{~nm}$, the measured photocurrent in the NIR can also not be explained by an enhanced light absorption in the bulk $a-\mathrm{Si}: \mathrm{H}$ $i$-layer. Photoexcitation of electrons within or in the vicinity of the NPs may explain the increased QE in the NIR. Figures 4(a) and 4(b) show schematic models of possible photoemission processes involved in the carrier-generation mechanism for the $\mathrm{Ag} / \mathrm{TCO} / \mathrm{NPs} / i / p / \mathrm{TCO}$ and $\mathrm{Ag} / \mathrm{TCO} / \mathrm{NPs} / i / n / \mathrm{TCO}$ structures, respectively. The $\mathrm{TCO} / \mathrm{NP} / i$ layer stack forms a Schottky contact. In case of the $\mathrm{Ag} / \mathrm{TCO} / \mathrm{NPs} / i / p / \mathrm{TCO}$ structure, electrons can be gen- 
erated by photoexcitation from the valence band of the $i$-layer into the TCO/NP contact. The $p$-type contact allows extraction of holes from the $i$-layer leading to a photocurrent. For the $\mathrm{Ag} / \mathrm{TCO} / \mathrm{NPs} / i / n / \mathrm{TCO}$, an electron can be generated by photoexcitation from the TCO/NP contact into the conduction band of the $i$-layer. The $n$-type contact allows extraction of electrons. For both structures, the photoexcitation of electrons can be enhanced by the LSPP-induced increase in the electromagnetic field within and in the surrounding of the NP. The $\mathrm{Ag} / \mathrm{TCO} / \mathrm{NPs} / i / n / \mathrm{TCO}$ structure clearly shows a larger enhancement of photocurrent in the NIR than the Ag/TCO/NPs/i/p/TCO structure. This difference may be explained by a difference in probability between excitation of electrons within the NP and excitation of electrons in the $i$-layer in close vicinity of the NP.

In order to verify that the photocurrent enhancement is actually caused by the LSPP effect in the NPs, a bulk-Ag/i/n/TCO layer stack was prepared. The Ag bulk layer consists of a $700 \mathrm{~nm} \mathrm{Ag} \mathrm{film} \mathrm{deposited} \mathrm{by} \mathrm{thermal}$ evaporation. Reflection measurements of the bulk-Ag/i/n/TCO layer stack show a slight decrease in the reflectivity in the long wavelength range [Fig. 2(b), dashed line] compared to the $\mathrm{Ag} / \mathrm{TCO} / \mathrm{i} / n / \mathrm{TCO}$ layer stack, probably due to absorption losses in the bulk Ag. ${ }^{13}$ The QE of the prepared layer stack exhibits a high signal in the short wavelength range due to interferences. However, no photocurrent is measured for $\lambda>750 \mathrm{~nm}$, as shown in Fig. 3(b) (dashed line). Thus, we conclude that the enhanced photocurrent in the NIR observed in the case of devices containing Ag NPs is caused by the resonant LSPP effect of the NPs.

In summary Ag NPs with radii below $20 \mathrm{~nm}$ were deposited by thermal evaporation and postannealing treatment at the TCO- $i$ interface of ultrathin $a-\mathrm{Si}: \mathrm{H}$ devices in the $\mathrm{Ag} / \mathrm{TCO} / i / n / \mathrm{TCO}$ and $\mathrm{Ag} / \mathrm{TCO} / i / p / \mathrm{TCO}$ configurations. Independently of the device configuration, an enhanced light absorption is observed in the long wavelength range for devices with NPs compared to devices without NPs, attributed to LSPP. The devices containing Ag NPs show an enhanced photocurrent in the NIR region of the spectrum. Since $a-\mathrm{Si}: \mathrm{H}$ only efficiently absorbs light for wavelengths up to $750 \mathrm{~nm}$, we assume that photoexcitation of electrons from the NPs or from states in close vicinity of the NPs at the LSPP resonance is responsible for the increased photocurrent. Theoretical investigations have to be made to verify the validity of the suggested explanation.

The authors like to acknowledge U. Kreibig, U. Rau, and U. Paetzold for constructive discussions and H. Siekmann, J. Klomfaß, and M. Hülsbeck for technical support.

${ }^{1}$ U. Kreibig and M. Vollmer, Optical Properties of Metal Clusters (Springer, Berlin, 1995).

${ }^{2}$ S. A. Maier, Plasmonics: Fundamentals and Applications (Springer, New York, 2007).

${ }^{3}$ D. Derkacs, S. H. Lim, P. Matheu, W. Mar, and E. T. Yu, Appl. Phys. Lett. 89, 093103 (2006).

${ }^{4}$ D. M. Schaadt, B. Feng, and E. T. Yu, Appl. Phys. Lett. 86, 063106 (2005).

${ }^{5}$ S. Pillai, K. R. Catchpole, T. Trupke, and M. A. Green, J. Appl. Phys. 101, 093105 (2007).

${ }^{6}$ E. Moulin, J. Sukmanowski, P. Luo, R. Carius, F. X. Royer, and H. Stiebig, J. Non-Cryst. Solids 354, 2488 (2008).

${ }^{7}$ M. Westphalen, U. Kreibig, J. Rostalski, H. Lüth, and D. Meissner, Sol. Energy Mater. Sol. Cells 61, 97 (2000).

${ }^{8}$ O. Stenzel, A. Stendal, K. Voigtsberger, and C. von Borczyskowski, Sol. Energy Mater. Sol. Cells 37, 337 (1995).

${ }^{9}$ C. Wen, K. Ishikawa, M. Kishima, and K. Yamada, Sol. Energy Mater. Sol. Cells 61, 339 (2000).

${ }^{10}$ C. Daboo, M. J. Baird, H. P. Hughes, N. Apsley, and T. Emeny, Thin Solid Films 201, 9 (1991).

${ }^{11}$ C. Sönnichsen, T. Franzl, T. Wilk, G. von Plessen, J. Feldmann, New J. Phys. 4, 93 (2002).

${ }^{12}$ C. Sönnichsen, S. Geier, N. E. Hecker, G. von Plessen, J. Feldmann, H. Ditlbacher, B. Lamprecht, J. R. Krenn, F. R. Aussenegg, V. Z.-H. Chan, J. P. Spatz, and M. Moeller, Appl. Phys. Lett. 77, 2949 (2000).

${ }^{13}$ J. Springer, A. Poruba, L. Müllerova, M. Vanecek, O. Kluth, and B. Rech, J. Appl. Phys. 95, 1427 (2004). 